\title{
Bending of an Infinite beam on a base with two parameters in the absence of a part of the base
}

\author{
Maxim Aleksandrovskiy ${ }^{1, *}$ and Lidiya Zaharova $^{1}$ \\ ${ }^{1}$ Moscow State University of Civil Engineering, Yaroslavskoe shosse, 26, Moscow, 129337, Russia
}

\begin{abstract}
Currently, in connection with the rapid development of high-rise construction and the improvement of joint operation of high-rise structures and bases models, the questions connected with the use of various calculation methods become topical. The rigor of analytical methods is capable of more detailed and accurate characterization of the structures behavior, which will affect the reliability of objects and can lead to a reduction in their cost. In the article, a model with two parameters is used as a computational model of the base that can effectively take into account the distributive properties of the base by varying the coefficient reflecting the shift parameter. The paper constructs the effective analytical solution of the problem of a beam of infinite length interacting with a two-parameter voided base. Using the Fourier integral equations, the original differential equation is reduced to the Fredholm integral equation of the second kind with a degenerate kernel, and all the integrals are solved analytically and explicitly, which leads to an increase in the accuracy of the computations in comparison with the approximate methods. The paper consider the solution of the problem of a beam loaded with a concentrated force applied at the point of origin with a fixed value of the length of the dip section. The paper gives the analysis of the obtained results values for various parameters of coefficient taking into account cohesion of the ground.
\end{abstract}

\section{Introduction}

Modern design and construction of high-rise buildings and structures are unthinkable without a clear understanding of the features of the work as well as a number of constructive elements of the future facility. At the same time, the issues related to the refinement and improvement of the parameters of the design schemes reflecting the interaction of structures with the base become urgent. The design schemes of unique objects, among which, first of all, are modern skyscrapers, meet the most stringent requirements in terms of their strength, reliability and durability. The uniqueness of high-rise buildings requires the improvement of calculation methods, taking into account the numerous factors of operational, technological and constructive nature. A significant part of the calculations related to the constructions of such objects is connected with the specification of efforts in the elements interacting with the base, and any refinement of calculations significantly affects the cost. This formulation of the problem fully relates to the tape foundations of high-rise buildings and structures, industrial

*Corresponding author: AleksandrovskiyMV@mgsu.ru 
and civil facilities, airfield and road surfaces, the operating conditions of which are associated with possible subsidence of the base and the formation of voids. When designing strip foundations located on karst rocks and subsidence soils in the form of loess deposits, which, when soaked, give a large draft, it is necessary to take into account the possibility of formation of depressions, dips, etc. under the foundations. The absence of contact of the beam with the base can occur at various sites, in particular, at the center or near the edge, which can be reduced to different statements of problems, for example, if the load and dip in the ground are in the central part of the strip foundation, then it can be calculated according to the scheme of infinite beams.

Despite the diversity of the computational models of bases and methods for calculating them, these methods are usually not interrelated and are used to solve particular problems. Among the elastic base models using two parameters, one can note the results presented in the works of M.M. Filonenko-Borodich [1], V.Z. Vlasova and N.N. Leontiev [2] and P.L. Pasternak [3]. The main advantage of such models was the ability to take into account the distributive capacity of the base. In the two-parameter model of the elastic base, the first coefficient characterizes the rigidity of the base by compression, and the second, by the shear. Despite the various ideas proposed for the calculation of beams on an elastic base, the authors come to the same kind of differential equation

$$
\frac{d^{4} w}{d x^{4}}-2 r^{2} \frac{d^{2} w}{d x^{2}}+s^{4} w=\frac{q a^{4}}{E_{1} J}
$$

where $r^{2}$ and $s^{2}$ are the generalized elastic dimensionless quantities, $a$ is a half-length of the beam.

Despite the availability of various techniques for determining the values of coefficients $[4,5,6]$ in the reports on engineering and geological surveys, as a rule, information on the above coefficients is absent. In SCAD Office in the Pasternak satellite program, the method proposed by M.I. Gorbunov-Posadov [7] is used as one of the possible.

In this formulation of the problem, the model proposed by V.Z. Vlasov, N.N. Leontiev [2] is used as a base, and the calculated values of the shear coefficient according to [8] are: $k=0, k=0.5$ и $k=0.9$.

The method of solving a differential equation of this kind was considered both on the basis of rigorous analytical methods, for example, [9, 10], and with the widely used, approximate numerical methods $[11,12]$.

\section{Materials and Methods}

To find the solution of the original differential equation, the method of generalized solutions proposed by V.I. Travush [10] is used. Later, this method was used to obtain an analytical solution to the bending of an infinite beam on a Winkler elastic foundation in the absence of a base under the beam part [13]. However, the main drawback of such a model of the basis is the lack of possibility to take into account its distributive capacity. The general form of the analytical solution for equation (1) was presented in [14], however, in our opinion, the authors made a number of inaccuracies, which in the case of implementing the above algorithm should lead to incorrect results.

Consider an infinite beam lying on an elastic base with two bed coefficients [2], and there is no base under the central part of the beam (Fig. 1.). 


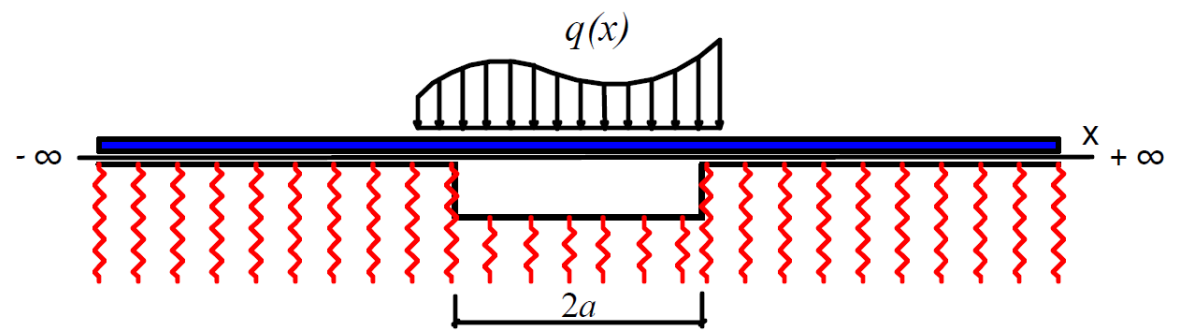

Fig.1. The scheme of an infinite beam lying on the base with two parameters in the absence of a base on a given section.

The arbitrary load $q(x)$, applied to the beam can be decomposed into symmetrical and inverse symmetric components, relative to the vertical axis of the beam. The differential equation describing the deflection of a beam for a given condition in the treatment of [2] takes the form

$$
E J y^{I V}(\xi)-2 t \theta\left(\xi-a_{1}\right) \cdot y^{\prime \prime}(\xi)+k \theta\left(\xi-a_{1}\right) y(\xi)=q(\xi)
$$

In the treatment [8], (10), the authors do not reflect the allowance for the absence of tangential stresses under that part of the beam where there is no base (see (13) [8]);

The equation (2) can be reduced to dimensionless coordinates by introducing the commonly used change of variables:

$$
\begin{gathered}
x=\xi \beta ; y(x)=y(\xi \beta) ; \theta(x-a)=\theta\left(\xi \beta-a_{1} \beta\right) ; k_{1}=\frac{2 t}{E J \beta^{2}}=\frac{4 t}{\sqrt{k E J}} ; \\
\frac{k}{E J}=4 \beta^{4} ; \beta=\left(\frac{k}{4 E J}\right)^{1 / 4} ; q_{0}(x)=\frac{1}{E J} q(x) .
\end{gathered}
$$

Here $E$ is the modulus of elasticity of the beam material; $J$ is its moment of inertia; $t$ and $k$ are the parameters of the base model; $k_{1}$ is the second coefficient of the bed; $\theta(x-a)$ is the Heaviside function

$$
\theta(x-a)= \begin{cases}0, & x<a \\ 1 . & x \geq a\end{cases}
$$

The differential equation for the deflection of a beam in dimensionless coordinates and functions, taking (2) into account, takes the form of

$$
y^{I V}(x)-k_{1} \theta(x-a) y^{\prime \prime}(x)+4 \theta(x-a) y(x)=4 k^{-1} q(x)
$$

To solve equation (4), we can use the Fourier transform to solve problems with an infinite domain, when the function $y(x)$ and its first three derivatives have no discontinuities in this area [15].

Under the action of the symmetric load component, applying the direct cosine of the Fourier transform separately for each term of equation (2), we obtain:

$$
\begin{aligned}
& \sqrt{\frac{2}{\pi}} \int_{0}^{\infty} y^{I V}(x) \cos \lambda x d x-k_{1} \sqrt{\frac{2}{\pi}}\left(\int_{0}^{\infty} y^{\prime \prime}(x) \cos \lambda x d x-\int_{0}^{a} y^{\prime \prime}(t) \cos \lambda t d t\right)+ \\
& +\sqrt{\frac{2}{\pi}} 4\left(\int_{0}^{\infty} y(x) \cos \lambda x d x-\int_{0}^{a} y(t) \cos \lambda t d t\right)=\sqrt{\frac{2}{\pi}} \frac{4}{k} \int_{0}^{\infty} q(x) \cos \lambda x d x
\end{aligned}
$$

Introducing the corresponding notation of Fourier transform:

$$
\sqrt{\frac{2}{\pi}} \int_{0}^{\infty} y^{I V}(x) \cos \lambda x d x=\lambda^{4} \sqrt{\frac{2}{\pi}} \int_{0}^{\infty} y(x) \cos \lambda x d x=\lambda^{4} Y(\lambda)
$$




$$
\begin{gathered}
\sqrt{\frac{2}{\pi}} \int_{0}^{\infty} y^{\prime \prime}(x) \cos \lambda x d x=-\lambda^{2} \sqrt{\frac{2}{\pi}} \int_{0}^{\infty} y(x) \cos \lambda x d x=-\lambda^{2} Y(\lambda) \\
\sqrt{\frac{2}{\pi}} \int_{0}^{\infty} q(x) \cos \lambda x d x=\sqrt{\frac{2}{\pi}} Q_{0}(\lambda)
\end{gathered}
$$

The general form of the equation with respect to a new dimensionless coordinate is

$$
Y(\lambda)=\frac{4}{k} \sqrt{\frac{2}{\pi}} \frac{Q_{0}(\lambda)}{\left(\lambda^{4}+k_{1} \lambda^{2}+4\right)}+\frac{\left(k_{1} \lambda^{2}+4\right)}{\left(\lambda^{4}+k_{1} \lambda^{2}+4\right)} \sqrt{\frac{2}{\pi}} \int_{0}^{a} y(t) \cos \lambda t d t
$$

In contrast to [8], here we take into account the change in the form of the cargo term on the right-hand side, due to the consideration of the distributive properties of the base.

Applying the inverse cosine of the Fourier transform to the resulting equation (6), we obtain an expression for the desired deflection function.

$$
y(x)=\frac{2}{\pi} \frac{4}{k} \int_{0}^{\infty} \frac{Q_{0}(\lambda)}{\left(\lambda^{4}+k_{1} \lambda^{2}+4\right)} \cos \lambda x d \lambda+\frac{2}{\pi} \int_{0}^{\infty} \frac{\left(k_{1} \lambda^{2}+4\right) \cos \lambda t \cos \lambda x}{\left(\lambda^{4}+k_{1} \lambda^{2}+4\right)} d \lambda \int_{0}^{a} y(t) d t
$$

Thus, applying the forward and inverse cosine of the Fourier transform to equation (2) allows us to reduce the differential equation (2) to the integral equation with respect to the sought deflection function of the beam $y(x)$.

$$
y(x)-\int_{0}^{a} y(t) K^{c}(x, t) d t=y(x)_{\infty}^{c}
$$

In this equation, the right-hand side $y(x)_{\infty}^{c}$ is the deflection of an infinite beam loaded with a given load and completely in contact with the two-parameter base along its entire length.

The general expression for the deflection function of an infinite beam for symmetric components $y(x)_{\infty}^{c}$, using [16] is written in the following form

$$
\begin{aligned}
& y(x)_{\infty}^{c}=\frac{2}{\pi} \frac{4}{k} \frac{P}{2} \beta \frac{1}{2}\left\{\frac { \pi } { 2 } \frac { 2 } { \sqrt { 1 6 - k _ { 1 } ^ { 2 } } } \frac { 1 } { 2 } e ^ { - | x _ { 0 } - x | \sqrt { 1 + \frac { k _ { 1 } } { 4 } } } \left(\sqrt{1-\frac{k_{1}}{4}} \cos \left(\left(x_{0}-x\right) \sqrt{1-\frac{k_{1}}{4}}\right)+\right.\right. \\
& \left.+\sqrt{1+\frac{k_{1}}{4}} \sin \left(\left|x_{0}-x\right| \sqrt{1-\frac{k_{1}}{4}}\right)\right)+\frac{\pi}{2} \frac{2}{\sqrt{16-k_{1}^{2}}} \frac{1}{2} e^{-\left|x_{0}+x\right| \sqrt{1+\frac{k_{1}}{4}}}\left(\sqrt{1-\frac{k_{1}}{4}} \cos \left(\left(x_{0}+x\right) \sqrt{1-\frac{k_{1}}{4}}\right)+\right. \\
& \left.\left.+\sqrt{1+\frac{k_{1}}{4}} \sin \left(\left(x_{0}+x\right) \sqrt{1-\frac{k_{1}}{4}}\right)\right)\right\}
\end{aligned}
$$

For the special case, when the load in the form of a concentrated force is applied in the center, i.e. $x_{0}=0$ the above expression takes the form of

$$
y(x)_{\infty}^{c}=\frac{2 P}{k} \beta \frac{1}{\sqrt{16-k_{1}^{2}}} e^{-|x| \sqrt{1+\frac{k_{1}}{4}}}\left(\sqrt{1-\frac{k_{1}}{4}} \cos \left(x \sqrt{1-\frac{k_{1}}{4}}\right)+\sqrt{1+\frac{k_{1}}{4}} \sin \left(|x| \sqrt{1-\frac{k_{1}}{4}}\right)\right)
$$

Introduce the notations:

$$
\begin{aligned}
& 1-\frac{k_{1}}{4}=A ; 1+\frac{k_{1}}{4}=B ;\left(4-\frac{k_{1}^{2}}{2}\right)=C ;-2 \sqrt{A}\left(4+k_{1}\right)=D ; 2 \sqrt{B}\left(4-k_{1}\right)=F ; \\
& 2 k_{1} \sqrt{A} \cdot \sqrt{B}=G ; \frac{2 \pi}{D^{2}+F^{2}}=H ; C F-G D=S ;[C D+G F]=-L .
\end{aligned}
$$

The use of the theory of residues makes it possible to express the kernel of the integral equation $(8) K^{c}(x, t)$ through a combination of trigonometric and hyperbolic functions:

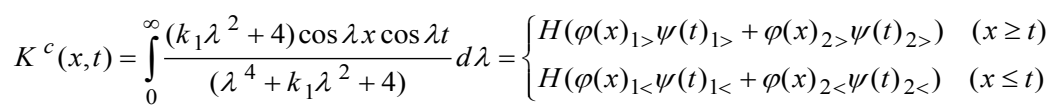

where 


$$
\begin{gathered}
\varphi(x)_{1>}=e^{-x \sqrt{B}} \cos (x \sqrt{A}), \varphi(x)_{2>}=e^{-x \sqrt{B}} \sin (x \sqrt{A}) \\
\psi(t)_{1>}=S \cos (t \sqrt{A}) \operatorname{ch}(t \sqrt{B})-L \sin (t \sqrt{A}) \operatorname{sh}(t \sqrt{B}) \\
\psi(t)_{2>}=S \sin (t \sqrt{A}) \operatorname{sh}(t \sqrt{B})+L \cos (t \sqrt{A}) \operatorname{ch}(t \sqrt{B}) \\
\varphi(x)_{1<}=\operatorname{ch}(x \sqrt{B}) \cos (x \sqrt{A}), \varphi(x)_{2<}=\operatorname{sh}(x \sqrt{B}) \sin (x \sqrt{A}), \\
\psi(t)_{1<}=(S \cos (t \sqrt{A})+L \sin (t \sqrt{A})) e^{-t \sqrt{B}}, \psi(t)_{2<}=(S \sin (t \sqrt{A})-L \cos (t \sqrt{A})) e^{-t \sqrt{B}} .
\end{gathered}
$$

It follows from (12) that the kernel of the integral equation (8) $-y(x)_{\infty}^{c}$ is degenerate and that, when solving it, we must consider two cases. The first of them assumes that the point at which the deflection is sought is in that part of the beam where there is a base beneath it. Obviously, in this case it follows from (8) and (12), an expression for determining the deflection function of a beam is

$$
y(x)^{c}=y(x)_{\infty}^{c}+H \sum_{i=1}^{2} c_{i>} \varphi(x)_{i>}
$$

here, the introduced notation is

$$
c_{i>}=\int_{0}^{a} y(t)^{c} \psi(t)_{i>} d t \quad(i=1,2)
$$

The coefficients $c_{i>}$ can be determined as a result of the standard procedure for solving integral equations with a degenerate kernel [5]. To this end, both sides of equation (14) are multiplied consecutively by $\psi(x)_{1>}$ and $\psi(x)_{2>}$, and then integrate from 0 to $a$.

The functions $\Phi_{i>k>}$ appearing in these expressions are integrals of combinations of functions $\varphi_{i>}$ and $\psi_{k>}$, the values of which can be expressed in explicit form.

$$
\begin{aligned}
& \Phi_{1>1>}=\int_{0}^{a} e^{-x \sqrt{B}} \cos (x \sqrt{A})(S \cos (x \sqrt{A}) \operatorname{ch}(x \sqrt{B})-L \sin (x \sqrt{A}) \operatorname{sh}(x \sqrt{B})) d x= \\
& =\frac{1}{2}\left\{S\left[\frac{\sin (2 a \sqrt{A})}{4 \sqrt{A}}+\frac{a}{2}+\frac{-e^{-2 a \sqrt{B}}(-\sqrt{A} \sqrt{B} \sin (2 a \sqrt{A})+B \cos (2 a \sqrt{A})+A+B)+A+2 B}{4 \sqrt{B}(A+B)}\right]-\right. \\
& \left.-L\left[\frac{\sin ^{2}(a \sqrt{A})}{2 \sqrt{A}}-\frac{\sqrt{A}-e^{-2 a \sqrt{B}}(\sqrt{B} \sin (2 a \sqrt{A})+\sqrt{A} \cos (2 a \sqrt{A}))}{4(A+B)}\right]\right\} \\
& \Phi_{1>2>}=\int_{0}^{a} e^{-x \sqrt{B}} \sin (x \sqrt{A})(S \cos (x \sqrt{A}) \operatorname{ch}(x \sqrt{B})-L \sin (x \sqrt{A}) \operatorname{sh}(x \sqrt{B})) d x= \\
& =\frac{1}{2}\left\{S\left[\frac{\sin ^{2}(a \sqrt{A})}{2 \sqrt{A}}+\frac{\sqrt{A}-e^{-2 a \sqrt{B}}(\sqrt{B} \sin (2 a \sqrt{A})+\sqrt{A} \cos (2 a \sqrt{A}))}{4(A+B)}\right]-\right. \\
& \left.-L\left[\frac{a}{2}-\frac{\sin ^{2}(2 a \sqrt{A})}{4 \sqrt{A}}-\frac{e^{-2 a \sqrt{B}}\left(A e^{2 a \sqrt{B}}-\sqrt{A} \sqrt{B} \sin (2 a \sqrt{A})+B \cos (2 a \sqrt{A})-A-B\right)}{4 \sqrt{B}(A+B)}\right]\right\} \\
& \quad \Phi_{2>1>}=\int_{0}^{a} e^{-x \sqrt{B}} \cos (x \sqrt{A})(S \sin (x \sqrt{A}) \operatorname{sh}(x \sqrt{B})+L \cos (x \sqrt{A}) \operatorname{ch}(x \sqrt{B})) d x= \\
& =\frac{1}{2}\left\{S\left[\frac{\sin ^{2}(a \sqrt{A})}{2 \sqrt{A}}-\frac{\sqrt{A}-e^{-2 a \sqrt{B}}(\sqrt{B} \sin (2 a \sqrt{A})+\sqrt{A} \cos (2 a \sqrt{A}))}{4(A+B)}\right]+\right. \\
& +L\left[\frac{\sin ^{2}(2 a \sqrt{A})}{4 \sqrt{A}}+\frac{a}{2}+\frac{-e^{-2 a \sqrt{B}}(-\sqrt{A} \sqrt{B} \sin (2 a \sqrt{A})+B \cos (2 a \sqrt{A})+A+B)+A+2 B}{4 \sqrt{B}(A+B)}\right.
\end{aligned}
$$




$$
\begin{aligned}
& \Phi_{2>2>}=\int_{0}^{a} e^{-x \sqrt{B}} \sin (x \sqrt{A})(S \sin (x \sqrt{A}) \operatorname{sh}(x \sqrt{B})+L \cos (x \sqrt{A}) \operatorname{ch}(x \sqrt{B})) d x= \\
& =\frac{1}{2}\left\{S\left[\frac{a}{2}-\frac{\sin (2 a \sqrt{A})}{4 \sqrt{A}}-\frac{e^{-2 a \sqrt{B}}\left(A e^{2 a \sqrt{B}}-\sqrt{A} \sqrt{B} \sin (2 a \sqrt{A})+B \cos (2 a \sqrt{A})-A-B\right)}{4 \sqrt{B}(A+B)}\right]+\right. \\
& \left.+L\left[\frac{\sin ^{2}(a \sqrt{A})}{2 \sqrt{A}}+\frac{\sqrt{A}-e^{-2 a \sqrt{B}}(\sqrt{B} \sin (2 a \sqrt{A})+\sqrt{A} \cos (2 a \sqrt{A}))}{4(A+B)}\right]\right\}
\end{aligned}
$$

For the case $x_{0}=0$ with allowance for (10) and (11) we obtain:

$$
\begin{aligned}
\Phi_{1 P>}= & \frac{2 P}{k} \beta \frac{1}{\sqrt{16-k_{1}^{2}}} \int_{0}^{a} e^{-|x| \sqrt{B}}(\sqrt{A} \cos (x \sqrt{A})+\sqrt{B} \sin (|x| \sqrt{A}))(S \cos (x \sqrt{A}) \operatorname{ch}(x \sqrt{B})-L \sin (x \sqrt{A}) \operatorname{sh}(x \sqrt{B})) d x= \\
= & \frac{P}{k} \beta \frac{2}{\sqrt{16-k_{1}^{2}}}\left(-\frac{\sqrt{A}(A+B)(-\sqrt{B} L-\sqrt{A} S)+\sqrt{B}\left(2 \sqrt{A} B L-3 A \sqrt{B} S-B^{3 / 2} S\right)}{8 \sqrt{A} \sqrt{B}(A+B)}+\right. \\
+ & \frac{1}{8 \sqrt{A} \sqrt{B}(A+B)} e^{-4 \sqrt{B}}\left(\sqrt{A}(A+B)\left(-4 B e^{4 \sqrt{B}} L-\sqrt{A} S-\sqrt{B}\left(L-4 \sqrt{A} e^{4 \sqrt{B}} S\right)\right)+\right. \\
+ & \sqrt{B}\left(A^{3 / 2}\left(-1+e^{4 \sqrt{B}}\right) L+\sqrt{A} B\left(1+e^{4 \sqrt{B}}\right) L-B^{3 / 2} e^{4 \sqrt{B}} S-A \sqrt{B}\left(2+e^{4 \sqrt{B}}\right) S\right) \cos (4 \sqrt{A})+ \\
+ & \sqrt{B}\left(B^{3 / 2} e^{4 \sqrt{B}} L+A \sqrt{B}\left(-2+e^{4 \sqrt{B}}\right) L+A \sqrt{B}\left(-1+e^{4 \sqrt{B}}\right) S+A^{3 / 2}\left(1+e^{4 \sqrt{B}}\right) S \sin (4 \sqrt{A})\right) \\
& \Phi_{2 P>}=\frac{2 P}{k} \beta \frac{1}{\sqrt{16-k_{1}^{2}}} \int_{0}^{a} e^{-|x| \sqrt{B}}(\sqrt{A} \cos (x \sqrt{A})+\sqrt{B} \sin (|x| \sqrt{A}))(S \sin (x \sqrt{A}) \operatorname{sh}(x \sqrt{B})+L \cos (x \sqrt{A}) \operatorname{ch}(x \sqrt{B})) d x= \\
= & \frac{P}{k} \beta \frac{2}{\sqrt{16-k_{1}^{2}}}\left(-\frac{\sqrt{A}(A+B)(-\sqrt{A} L+\sqrt{B} S)-\sqrt{B}\left(3 A \sqrt{B} L+2 \sqrt{A} B S+B^{3 / 2} L\right)}{8 \sqrt{A} \sqrt{B}(A+B)}\right. \\
& +\frac{1}{8 \sqrt{A} \sqrt{B}(A+B)} e^{-4 \sqrt{B}}\left(\sqrt{A}(A+B)\left(\sqrt{A}\left(-1+4 \sqrt{B} e^{4 \sqrt{B}}\right) L+\sqrt{B} S+4 B e^{4 \sqrt{B}} S\right)-\sqrt{B}\left(B^{3 / 2} e^{4 \sqrt{B}} L+\right.\right. \\
& \left.+A \sqrt{B}\left(2+e^{4 \sqrt{B}}\right) L+A^{3 / 2}\left(-1+e^{4 \sqrt{B}}\right) S+\sqrt{A} B\left(1+e^{4 \sqrt{B}}\right) S\right) \cos (4 \sqrt{A})+\sqrt{B}\left(\sqrt{A} B\left(-1+e^{4 \sqrt{B}}\right) L+\right. \\
& \left.\left.\left.+A^{3 / 2}\left(1+e^{4 \sqrt{B}}\right) L-B^{3 / 2} e^{4 \sqrt{B}} S-A \sqrt{B}\left(-2+e^{4 \sqrt{B}}\right) S\right) \sin (4 \sqrt{A})\right)\right)
\end{aligned}
$$

After determining the coefficients $c_{i>}$, the deflection function is known, hence the expressions for the angles of rotation, bending moments and transverse forces on the section of the beam in question are also known. We give their real values for the case of a concentrated force applied at the center of the beam:

$$
\begin{gathered}
y(x)^{c}=\frac{P}{2 E J \beta^{3}} \frac{1}{\sqrt{16-k_{1}^{2}}}\left[e^{-x x \mid \sqrt{B}}(\sqrt{A} \cos (x \sqrt{B})+\sqrt{B} \sin (|x| \sqrt{A}))+\right. \\
\left.+C_{1>} e^{-x \sqrt{B}} \cos (x \sqrt{A})+C_{2>} e^{-x \sqrt{B}} \sin (x \sqrt{A})\right] ; \\
\varphi(x)^{c}=\frac{P}{2 E J \beta^{2}} \frac{1}{\sqrt{16-k_{1}^{2}}}\left[-\sqrt{B} C_{1>} e^{-x \sqrt{B}} \cos (x \sqrt{A})+\sqrt{A} C_{2>} e^{-x \sqrt{B}} \cos (x \sqrt{A})-\right. \\
-\sqrt{A} C_{1>} e^{-x \sqrt{B}} \sin (x \sqrt{A})-\sqrt{B} C_{2>} e^{-x \sqrt{B}} \sin (x \sqrt{A})+e^{-x \sqrt{B}}(\sqrt{A} \sqrt{B} \cos (x \sqrt{A})- \\
\left.-A \sin (x \sqrt{A}))-\sqrt{B} e^{-x \sqrt{B}}(\sqrt{A} \cos (x \sqrt{A})+\sqrt{B} \sin (x \sqrt{A}))\right] ; \\
M(x)^{c}=-\frac{P}{2 \beta} \frac{1}{\sqrt{16-k_{1}^{2}}}\left[(A+B) e^{-x \sqrt{B}}(-\sqrt{A} \cos (x \sqrt{A})+\sqrt{B} \sin (x \sqrt{A}))+\right. \\
+C_{1>} e^{-x \sqrt{B}}[(-A+B) \cos (x \sqrt{A})+2 \sqrt{A} \sqrt{B} \sin (x \sqrt{A})]+ \\
\left.+C_{2>} e^{-x \sqrt{B}}[-2 \sqrt{A} \sqrt{B} \cos (x \sqrt{A})+(-A+B) \sin (x \sqrt{A})]\right] ;
\end{gathered}
$$




$$
\begin{aligned}
& Q(x)^{c}=-\frac{P}{2} \frac{1}{\sqrt{16-k_{1}^{2}}}\left[3 A \sqrt{B} C_{1>} e^{-x \sqrt{B}} \cos (x \sqrt{A})-B^{3 / 2} C_{1>} e^{-x \sqrt{B}} \cos (x \sqrt{A})-\right. \\
& -A^{3 / 2} C_{2} e^{-x \sqrt{B}} \cos (x \sqrt{A})+3 \sqrt{A} B C_{2>} e^{-x \sqrt{B}} \cos (x \sqrt{A})+A^{3 / 2} C_{1>} e^{-x \sqrt{B}} \sin (x \sqrt{A})- \\
& -3 \sqrt{A} B C_{1>} e^{-x \sqrt{B}} \sin (x \sqrt{A})+3 A \sqrt{B} C_{2>} e^{-x \sqrt{B}} \sin (x \sqrt{A})-B^{3 / 2} C_{2>} e^{-x \sqrt{B}} \sin (x \sqrt{A})+ \\
& +3 B e^{-x \sqrt{B}}(\sqrt{A} \sqrt{B} \cos (x \sqrt{A})-A \sin (x \sqrt{A}))+e^{-x \sqrt{B}}\left(-A^{3 / 2} \sqrt{B} \cos (x \sqrt{A})+\right. \\
& \left.+A^{2} \sin (x \sqrt{A})\right)-B^{3 / 2} e^{-x \sqrt{B}}(\sqrt{A} \cos (x \sqrt{A})+\sqrt{B} \sin (x \sqrt{A}))- \\
& \left.-3 \sqrt{B} e^{-x \sqrt{B}}\left(-A^{3 / 2} \cos (x \sqrt{A})-A \sqrt{B} \sin (x \sqrt{A})\right)\right] .
\end{aligned}
$$

It is more difficult to determine the deflection function in the beam portion where there is no base, i. e. $-a \leq x \leq a$. In this case we obtain from (8) and (12)

$$
y(x)^{c}=y(x)_{\infty}^{c}+H \sum_{i=1}^{2}\left[\varphi(x)_{i>} \int_{0}^{x} y(t)^{c} \psi(t)_{i>} d t+\varphi(x)_{i<} \int_{x}^{a} y(t)^{c} \psi(t)_{i<} d t\right]
$$

The solution of this equation can be obtained, for example, by the method of successive approximations; the corresponding integrals are calculated in explicit form.

$$
\begin{gathered}
y(x)^{c}=y(x)_{\infty}^{c}+H \sum_{i=1}^{2}\left[\varphi(x)_{i>} \int_{0}^{x} y(t)^{c} \psi(t)_{i>} d t+\varphi(x)_{i<} \int_{x}^{a} y(t)^{c} \psi(t)_{i<} d t\right]= \\
=H\left[\varphi(x)_{1>} \int_{0}^{x} y(t)^{c} \psi(t)_{1>} d t+\varphi(x)_{1<} \int_{x}^{a} y(t)^{c} \psi(t)_{1<} d t+\varphi(x)_{2>} \int_{0}^{x} y(t)^{c} \psi(t)_{2>} d t+\varphi(x)_{2<} \int_{x}^{a} y(t)^{c} \psi(t)_{2<} d t\right]+y(x)_{\infty}^{c} \\
C_{1}=\int_{0}^{x} y(t)^{c} \psi(t)_{1>} d t ; \quad C_{2}=\int_{x}^{a} y(t)^{c} \psi(t)_{1<} d t ; \quad C_{3}=\int_{0}^{x} y(t)^{c} \psi(t)_{2>} d t ; \quad C_{4}=\int_{x}^{a} y(t)^{c} \psi(t)_{2<} d t \\
y(x)^{c}=C_{1} \varphi(x)_{1>}+C_{2} \varphi(x)_{1<}+C_{3} \varphi(x)_{2>}+C_{4} \varphi(x)_{2<}+y(x)_{\infty}^{c}
\end{gathered}
$$

Substitute (21) in (20)

$$
\begin{aligned}
& C_{1}=\int_{0}^{x} y(t)^{c} \psi(t)_{1>} d t=\int_{0}^{x}\left(C_{1} \varphi(t)_{1>}+C_{2} \varphi(t)_{1<}+C_{3} \varphi(t)_{2>}+C_{4} \varphi(t)_{2<}+y(t)_{\infty}^{c}\right) \psi(t)_{1>} d t \\
& C_{2}=\int_{x}^{a} y(t)^{c} \psi(t)_{1<} d t=\int_{x}^{a}\left(C_{1} \varphi(t)_{1>}+C_{2} \varphi(t)_{1<}+C_{3} \varphi(t)_{2>}+C_{4} \varphi(t)_{2<}+y(t)_{\infty}^{c}\right) \psi(t)_{1<} d t \\
& C_{3}=\int_{0}^{x} y(t)^{c} \psi(t)_{2>} d t=\int_{0}^{x}\left(C_{1} \varphi(t)_{1>}+C_{2} \varphi(t)_{1<}+C_{3} \varphi(t)_{2>}+C_{4} \varphi(t)_{2<}+y(t)_{\infty}^{c}\right) \psi(t)_{2>} d t \\
& C_{4}=\int_{x}^{a} y(t)^{c} \psi(t)_{2<} d t=\int_{x}^{a}\left(C_{1} \varphi(t)_{1>}+C_{2} \varphi(t)_{1<}+C_{3} \varphi(t)_{2>}+C_{4} \varphi(t)_{2<}+y(t)_{\infty}^{c}\right) \psi(t)_{2<} d t .
\end{aligned}
$$

We give the form of the calculated values of the first four integrals representing the corresponding coefficients:

$$
\begin{aligned}
& f_{11}=H \int_{0}^{x} \varphi(t)_{1>} \psi(t)_{1>} d t=\frac{H}{4 \pi}\left[S\left(\frac{1}{\sqrt{B}}+\frac{\sqrt{B}}{A+B}-\frac{e^{-2 \sqrt{B} x}}{\sqrt{B}}+2 x+\frac{e^{-2 \sqrt{B} x}\left(-\sqrt{A} \sqrt{B} \cos (2 \sqrt{A} x)+\left(A+A e^{2 \sqrt{B} x}+B e^{2 \sqrt{B} x}\right) \sin (2 \sqrt{A} x)\right)}{\sqrt{A}(A+B)}\right)-\right. \\
& \left.-L\left(\frac{\left(-1+\frac{A e^{-2 \sqrt{B} x}}{A+B}\right) \cos (2 \sqrt{A} x)}{\sqrt{A}}+\frac{\frac{B}{\sqrt{A}}+\sqrt{B} e^{-2 \sqrt{B} x} \sin (2 \sqrt{A} x)}{A+B}\right)\right]
\end{aligned}
$$

$f_{12}=H \int_{0}^{x} \varphi(t)_{1<} \psi(t)_{1>} d t=\frac{H}{4 \sqrt{A} \sqrt{B}(A+B) \pi}[\sqrt{A} \sqrt{B}(-L \sqrt{B}+S \sqrt{A}) \operatorname{ch}[2 \sqrt{B} x] \sin (2 \sqrt{A} x)+\sqrt{B}(A+B) S(2 \sqrt{A} x+\sin (2 \sqrt{A} x))+$ $+\sqrt{A}((A+B) S+(\sqrt{A} \sqrt{B} L+S B) \cos (2 \sqrt{A} x) \operatorname{sh}[2 \sqrt{B} x]]$

$$
\begin{aligned}
& f_{13}=H \int_{0}^{x} \varphi(t)_{2>} \psi(t)_{1>} d t=\frac{H}{4 \pi}\left[S\left(\frac{1}{\sqrt{A}}+\frac{\sqrt{A}}{A+B}-\frac{\cos (2 \sqrt{A} x)}{\sqrt{A}}-\frac{e^{-2 \sqrt{B} x}(\sqrt{A} \cos (2 \sqrt{A} x)+B \sin (2 \sqrt{A} x))}{(A+B)}\right)-\right. \\
& \left.-L\left(-\frac{1}{\sqrt{B}}+\frac{\sqrt{B}}{A+B}+\frac{e^{-2 \sqrt{B} x}}{\sqrt{B}}+2 x-\frac{e^{-2 \sqrt{B} x}\left(\sqrt{A} \sqrt{B} \cos (2 \sqrt{A} x)+\left(B e^{2 \sqrt{B} x}+A\left(-1+e^{2 \sqrt{B} x}\right)\right) \sin (2 \sqrt{A} x)\right)}{\sqrt{A}(A+B)}\right)\right]
\end{aligned}
$$




$$
\begin{aligned}
& f_{14}=H \int_{0}^{x} \varphi(t)_{1<} \psi(t)_{1>} d t=\frac{H}{4 \sqrt{A} \sqrt{B}(A+B) \pi}[\sqrt{B}(A+B) L(2 \sqrt{A} x-\sin (2 \sqrt{A} x))+ \\
& +\sqrt{A} \sqrt{B}(L \sqrt{A}+S \sqrt{B}) c h[2 \sqrt{B} x] \sin (2 \sqrt{A} x)-\sqrt{A}((A+B) L+(-B L+\sqrt{A} \sqrt{B} S) \cos (2 \sqrt{A} x)) \operatorname{sh}[2 \sqrt{B} x]] \\
& f_{1 p}=\int_{0}^{x} y(t)_{\infty}^{c} \psi(t)_{1>} d t=\frac{1}{16 \sqrt{A} \sqrt{B}(A+B) \sqrt{16-k^{2}}[-\sqrt{A}(A+B)(-L \sqrt{B}-S \sqrt{A})+} \\
& +\sqrt{B}\left(2 \sqrt{A} B L-3 A \sqrt{B} S-B^{3 / 2} S\right)+e^{-2 \sqrt{B} x}\left(\sqrt{A}(A+B)\left(-S \sqrt{A}-2 B e^{2 \sqrt{B} x} L x-\sqrt{B}\left(L-2 \sqrt{A} e^{2 \sqrt{B} x} S x\right)\right)+\right. \\
& \sqrt{B}\left(A^{3 / 2}\left(-1+e^{2 \sqrt{B} x}\right) L+\sqrt{A} B\left(1+e^{2 \sqrt{B} x}\right) L-B^{3 / 2} e^{2 \sqrt{B} x} S-A \sqrt{B}\left(2+e^{2 \sqrt{B} x}\right) S\right) \cos (2 \sqrt{A} x)+ \\
& \left.\left.+\sqrt{B}\left(B^{3 / 2} e^{2 \sqrt{B} x} L+\sqrt{A} B\left(-2+e^{2 \sqrt{B} x}\right) L+\sqrt{A} B\left(-1+e^{2 \sqrt{B} x}\right) S+A^{3 / 2}\left(1+e^{2 \sqrt{B} x}\right) S\right) \sin (2 \sqrt{A} x)\right)\right]
\end{aligned}
$$

The expression determining the values of the bending moment at the section where the base under the beam is absent will take the following form

$$
\begin{aligned}
& M(x)^{c}=-\frac{P}{2 \beta} \frac{1}{\sqrt{16-k_{1}^{2}}}\left[(A+B) e^{-x \sqrt{B}}(-\sqrt{A} \cos (x \sqrt{A})+\sqrt{B} \sin (x \sqrt{A}))\right]+ \\
& +C_{1<} e^{-x \sqrt{B}}[(-A+B) \cos (x \sqrt{A})+2 \sqrt{A} \sqrt{B} \sin (x \sqrt{A})]-C_{2<}[(A-B) \cos (x \sqrt{A}) \operatorname{ch}(x \sqrt{B})+ \\
& +2 \sqrt{A} \sqrt{B} \sin (x \sqrt{A}) \operatorname{sh}(x \sqrt{B})]+C_{3<} e^{-x \sqrt{B}}[-2 \sqrt{A} \sqrt{B} \cos (x \sqrt{A})+(-A+B) \sin (x \sqrt{A})]+ \\
& +C_{4<}[2 \sqrt{A} \sqrt{B} \cos (x \sqrt{A}) \operatorname{ch}(x \sqrt{B})+(-A+B) \sin (x \sqrt{A}) \operatorname{sh}(x \sqrt{B})]
\end{aligned}
$$

\section{Results}

As an example of using the obtained dependences, we give the calculation of an infinite beam, under the central part of which there is no base for the given section $2 a=1$. The beam is loaded in the center by concentrated force $P$. The diagrams of these deflections and bending moments are shown in Figure 2 and Figure 3, for different values of the coefficient of adhesion. In the same place, the corresponding diagrams for an infinite beam everywhere contacting the base are shown in dashed lines. Actual values of deformations and forces can be obtained using (17), (18), and (24).

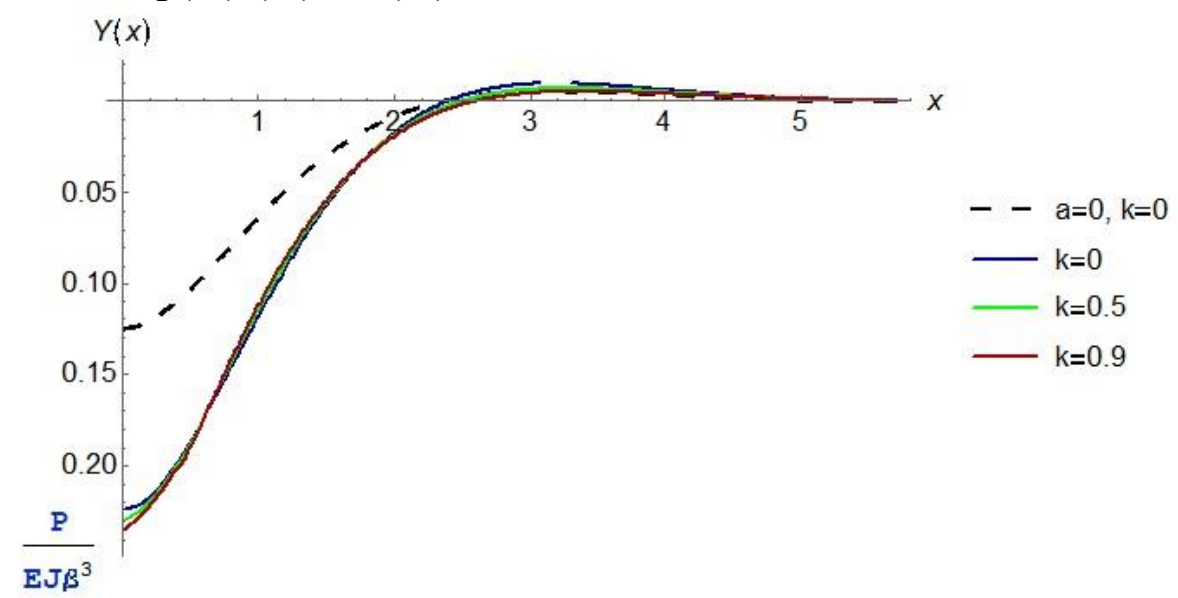

Fig.2. Diagrams of an infinite beam deflections with $a=0.5$ and $k=0, k=0.5, k=0.9$. 


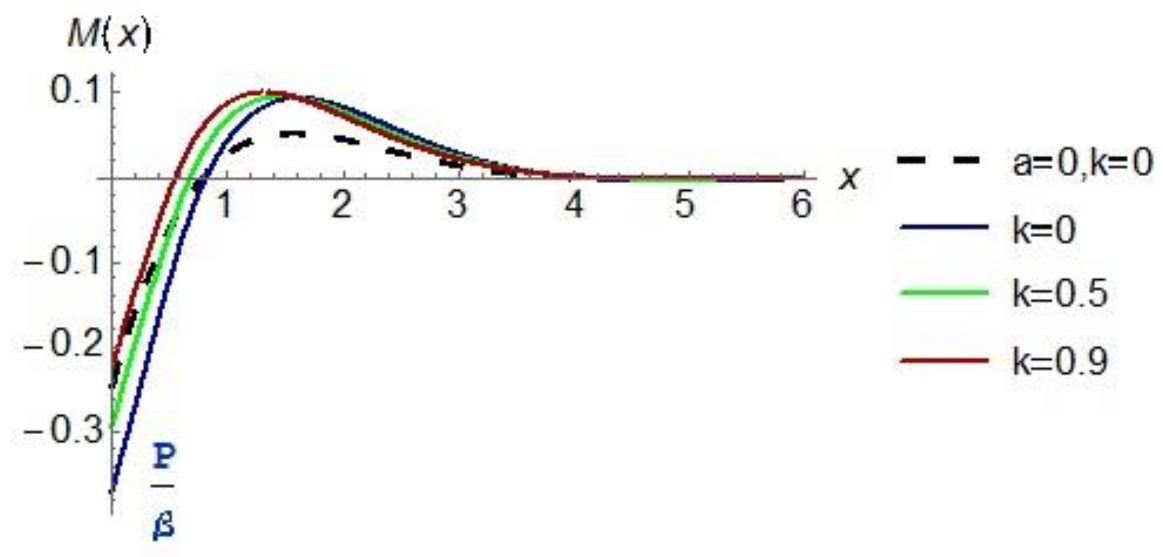

Fig.3. The bending moment diagrams of an infinite beam with $a=0.5$ and $k=0, k=0.5$, $k=0.9$.

When a skew-symmetric load acts on the beam, the application of the sine-transformation to differential equation (4) makes it possible to reduce it to an integral equation of the type (12), but the right side of it will be the deflection function of an infinite beam from a given skew-symmetric load $y(x)_{\infty}^{s}$, and the kernel of the equation is the function

$$
K^{s}(x, t)=\int_{0}^{\infty} \frac{\left(k_{1} \lambda^{2}+4\right) \sin \lambda x \sin \lambda t}{\left(\lambda^{4}+k_{1} \lambda^{2}+4\right)} d \lambda= \begin{cases}H\left\{\varphi(x)_{1>} \psi(t)_{1>}+\varphi(x)_{2>} \psi(t)_{2>}\right\} & (x \geq t) \\ H\left\{\varphi(x)_{1<} \psi(t)_{1<}+\varphi(x)_{2<} \psi(t)_{2<}\right\} & (x \leq t)\end{cases}
$$

Here the functions:

$$
\begin{gathered}
\varphi(x)_{1>}=e^{-x \sqrt{B}} \cos (x \sqrt{A}), \varphi(x)_{2>}=e^{-x \sqrt{B}} \sin (x \sqrt{A}), \\
\psi(t)_{1>}=S \cos (t \sqrt{A}) \operatorname{sh}(t \sqrt{B})-L \sin (t \sqrt{A}) \operatorname{ch}(t \sqrt{B}) \\
\psi(t)_{2>}=S \sin (t \sqrt{A}) \operatorname{ch}(t \sqrt{B})+L \cos (t \sqrt{A}) \operatorname{sh}(t \sqrt{B}) \\
\varphi(x)_{1<}=\operatorname{sh}(x \sqrt{B}) \cos (x \sqrt{A}), \varphi(x)_{2<}=\operatorname{ch}(x \sqrt{B}) \sin (x \sqrt{A}), \\
\psi(t)_{1<}=(S \cos (t \sqrt{A})+L \sin (t \sqrt{A})) e^{-t \sqrt{B}}, \psi(t)_{2<}=(S \sin (t \sqrt{A})-L \cos (t \sqrt{A})) e^{-t \sqrt{B}} .
\end{gathered}
$$

\section{Conclusions}

The perfection of the behavior simulation of an infinite beam lying on a base determined by two parameters and having a dip under the loaded part is of great importance for the design of high-rise buildings and structures. Taking into account the dips in the ground significantly changes the values of the calculated deformations and forces in the beam. The study can be used to analyze the strength and practical design, for example, of strip foundations. The analysis of the results obtained using different values of the coefficients showed that the proposed calculation method and the model of the base under consideration are simple and convenient for practical calculations and can constitute an alternative to other engineering methods. The proposed method of calculation can be easily extended to cases where the dip occurs not under one, but under several sections of the beam simultaneously.

\section{References}

1. M. Filonenko-Borodich Collection of papers MEMIIT, Issue 53. (1945) 
2. V. Vlasov, et al. Beams, Plates and Shells on Elastic Foundation. - Moscow: Fizmatgiz, p. 75 (1960)

3. P. Pasternak Fundamentals of a New Method for Calculating Foundations on Elastic Foundation with the Help of Two Bed Coefficients. - M. - L .: Gosstroyizdat, p. 56 (1964)

4. A. Kerr, On the formal development of elastic foundation models, Ing.-Arc., (1984)

5. S. Kolyasina, et al. The electronic scientific publication "Scientists' Notes PNU", 5, 2, pp. $21-34,(2014)$

6. C. Vallabhan, et al. An improved model for beams on elastic foundations, Elastic-Plastic Failure Modelling of Structures with Applications Presented at the (1988)

7. M. Gorbunov-Posadov, et al. Calculation of Structures on an Elastic Foundation / M.I. Gorbunov-Posadov, (Moscow, Stroyizdat, 1994)

8. V. Travush, et al. Bending of Uninsulated Rectangular Slabs Lying on a Two-parameter Base (Izv. universities. Stroitelstvo, 1998)

9. D. Dobromir Engineering Mechanics, 19, No. 6, p. 381-392, (2012)

10. V. Travush V.I. The Method of Generalized Solutions in the Problems of Bending of Slabs on a Linearly Deformable Bed / Stroitelnaya Mechanica i Raschet Sooruzheniy. 1, 24-28 (1982)

11. G. Wei , A New Algorithm for Solving some Mechanical Problems, Computer Methods in Applied Mechanics and Engineering, 190,2017-2030, (2001)

12. G. Wei, Discrete Singular Convolution and its Application to the Analysis of Plates with Internal Supports. Part 1: Theory and algorithm International Journal for Numerical Methods in Engineering, 55,913-946, (2002)

13. V. Travush II Republican Scientific and Technical Conference. Scientific and technological progress and ecology (Aktau, 1992)

14. A. Marufiy, Vestnik KSUCTA 1 pp.252-256 (2016)

15. I. Geldfand I.M., Shilov G.E. Generalized Functions and Actions over Them (Moscow, Dobrosvet (2007)

16. I. Gradshtein Tables of Integrals, Series and Products (St. Petersburg, BHVPetersburg,2011) 